University of Wollongong

Research Online

Faculty of Social Sciences - Papers (Archive) Faculty of Arts, Social Sciences \& Humanities

$1-1-2020$

\title{
A Systematic Review of Parent and Caregiver Mental Health Literacy
}

Diarmuid Hurley

University of Wollongong, dsh725@uowmail.edu.au

Christian Swann

Mark S. Allen

University of Wollongong, markal@uow.edu.au

Helen L. Ferguson

University of Wollongong

Stewart A. Vella

University of Wollongong, stvella@uow.edu.au

Follow this and additional works at: https://ro.uow.edu.au/sspapers

Part of the Education Commons, and the Social and Behavioral Sciences Commons

Research Online is the open access institutional repository for the University of Wollongong. For further information contact the UOW Library: research-pubs@uow.edu.au 


\title{
A Systematic Review of Parent and Caregiver Mental Health Literacy
}

\author{
Abstract \\ 2019, Springer Science+Business Media, LLC, part of Springer Nature. This study aimed to systematically \\ review the current body of research on parent and caregiver mental health literacy. Electronic databases \\ were searched in January 2018 with 21 studies meeting inclusion criteria. A narrative synthesis of \\ quantitative and qualitative studies was conducted. Findings across studies suggest that parents and \\ caregivers had limited mental health knowledge. Factors associated with help-seeking included cultural \\ and religious beliefs, financial and knowledge barriers, fear and mistrust of treatment services, and \\ stigma. Notable limitations include non-representative samples, cross-sectional research designs, and \\ use of inconsistent and non-validated study measures. Research would benefit from more diverse \\ samples, an increased focus on prevention, and controlled trials of educational programmes targeting \\ mental health literacy. \\ Disciplines \\ Education | Social and Behavioral Sciences \\ Publication Details \\ Hurley, D., Swann, C., Allen, M., Ferguson, H. L. \& Vella, S. (2020). A Systematic Review of Parent and \\ Caregiver Mental Health Literacy. Community Mental Health Journal, 56 (2),
}


Running head: PARENT AND CAREGIVER MENTAL HEALTH LITERACY

A Systematic Review of Parent and Caregiver Mental Health Literacy 


\begin{abstract}
This study aimed to systematically review the current body of research on parent and caregiver mental health literacy. Electronic databases were searched in January 2018 with 21 studies meeting inclusion criteria. A narrative synthesis of quantitative and qualitative studies was conducted. Findings across studies suggest that parents and caregivers had limited mental health knowledge. Factors associated with help-seeking included cultural and religious beliefs, financial and knowledge barriers, fear and mistrust of treatment services, and stigma. Notable limitations include non-representative samples, cross-sectional research designs, and use of inconsistent and non-validated study measures. Research would benefit from more diverse samples, an increased focus on prevention, and controlled trials of educational programmes targeting mental health literacy.
\end{abstract}

Keywords: child and adolescent mental health; help-seeking; mental health knowledge; mental health attitudes; narrative synthesis 
A Systematic Review of Parent and Caregiver Mental Health Literacy

\section{Introduction}

Mental health literacy refers to knowledge, attitudes, and beliefs about mental health disorders, helpseeking and treatment options that aid in the recognition, management, and prevention of such disorders (Jorm, 2012; Jorm et al., 1997). It encapsulates the knowledge of professional help-seeking and treatment options, the ability to recognise symptoms of mental health disorders, and the capacity and willingness to effectively support and assist others experiencing symptoms (Jorm et al., 1997). The conceptualisation of mental health literacy appears to align closely with the concepts of both health literacy and health activation. While health literacy focuses predominantly on the skills involved in managing health outcomes (e.g. knowing where or how to seek help), patient activation additionally encompasses an individual's self-efficacy and motivation to take action to manage one's healthcare and well-being (Hibbard, 2017; Hibbard \& Gilburt, 2014).

Previous experience of mental illness and treatment utilisation is associated with better mental health literacy (Cutler, Reavley, \& Jorm, 2017; Mendenhall \& Frauenholtz, 2013). Females have been found to be more mental health literate than males (Mendenhall \& Frauenholtz, 2013), with Western populations demonstrating better mental health literacy than other populations, especially those in the developing world (Furnham \& Hamid, 2014). However, a significant percentage of the population either struggle to spot the signs of a mental health disorder or are uncertain about how to respond if faced with a mental health disorder (Jorm, 2012; Jorm, et al., 1997). This includes parents and caregivers of adolescents who report not knowing how or when to intervene (Boulter \& Rickwood, 2013). In Australia, approximately $75 \%$ of the public who had contact with a family member or friend experiencing symptoms of a mental health disorder have attempted to provide help and support (Reavley \& Jorm, 2012). This finding emphasises the need for widespread mental health literacy education, including how best to respond and assist in a mental health crisis. This is especially important in regards to educating those adults in a position of care and responsibility on how to prevent and manage youth mental health disorders (Kelly et al., 2011)

Relatively little is known about the factors that influence parental mental health literacy (Frauenholtz, Conrad-Hiebner, \& Mendenhall, 2015). Less still is known about the mental health literacy of those others in a position of direct responsibility for children and adolescents, such as family caregivers, legal guardians and foster carers. Moreover, the relationship between parents' health activation and their confidence and ability to seek help for others is unclear. This is surprising, considering the prevalence of child and adolescent mental health disorders, and the importance of parent and caregiver mental health literacy for child and adolescent 
mental health outcomes. During childhood and adolescence, approximately $10-20 \%$ of the global population are affected by mental health disorders (Kieling et al., 2011), with a recent meta-analysis estimating a pooled worldwide prevalence of 13.4\% (Polanczyk et al., 2015). However, a significant treatment gap exists for children and adolescents (Gulliver, Griffiths, \& Christensen, 2010).

Adolescents identify their parents as a primary source of support (Jorm \& Wright, 2007) and are more likely to seek and receive professional help for mental health disorders if recommended and supported by their family (Rickwood, Deane, \& Wilson, 2007). Indeed, parents have reported that one of their main responsibilities in managing their adolescent's mental illness is to encourage and facilitate appropriate treatment (Honey, Alchin, \& Hancock, 2014). Importantly, a recent systematic review by Reardon et al. (2017) identified barriers and facilitators to accessing youth mental health services across parent knowledge, understanding and help-seeking attitudes. Prominent barriers for parents include: recognition of a problem and need to help; lack of knowledge of help-seeking options; stigma; trust in mental health professionals; time; and, cost. Inadequate mental health literacy among parents and caregivers can have detrimental consequences for child and adolescent mental health including delayed help seeking, lack of treatment, and higher levels of stigma (Gronholm et al., 2015; Mendenhall, 2012). In addition, poor health activation is associated with a lack of help-seeking and treatment utilisation (Hibbard \& Gilburt, 2014).

Mental health literacy can be improved at individual, community, and population levels through face to face intervention, mass media campaigns, and via self-directed online channels (Jorm, 2012). Individuals, groups and communities participating in mental health literacy campaigns are assumed to increase their knowledge and confidence to help others, reduce stigmatising attitudes, and engage in actual help-seeking and support strategies (Anderson \& Pierce, 2012). Reviews of mental health literacy interventions have shown improvements in knowledge, help-seeking attitudes and supportive behaviours (Brijnath et al., 2016;; Morgan, Ross, \& Reavley, 2018), but have not significantly influenced actual help-seeking behaviours (Gulliver, Griffiths, Christensen, \& Brewer, 2012). In addition, health activation can be modified through intervention (Greene \& Hibbard, 2012), and interventions to increase patient activation have resulted in more positive attitudes towards help-seeking and treatment, increased engagement and retention in mental health programs, and a reduction in mental health disorder symptomology (Alegria et al., 2013; Green et al., 2010).

To date, no systematic review has examined the literature on mental health literacy as it relates to parents and other caregivers of children and adolescents. The purpose of the current review was to collate, synthesise, and evaluate the available research on parent and caregiver mental health literacy. Moreover, this 
review aimed to synthesise what is known about parent and caregiver mental health knowledge, attitudes and help-seeking practices as it pertains to child and adolescent mental health.

\section{Method}

This systematic review used the PRISMA-P guidelines for preferred reporting items for systematic reviews and meta-analyses (Moher et al., 2015).

\section{Search Strategy}

A search strategy was developed over multiple iterations by the research team, with initial search terms created by examining the concept of mental health literacy and its various components (Jorm et al., 1997). Search terms used in previous systematic reviews examining mental health literacy (e.g., Brijnath et al., 2016) were also examined. Preliminary search returns were then mined (Weed, Coren, \& Fiore, 2009) for consistent, alternative key words, to identify the most appropriate and comprehensive search terms. During this process it became evident that the inclusion of some independent search phrases such as "mental health" yielded excessive search returns and so were excluded. "Mental health literacy" was chosen as an encompassing search term likely to capture parent or caregiver knowledge, attitudes and help-seeking practices in relation to mental health. Subcomponents of mental health literacy (e.g., mental health knowledge) were also included to optimise relevant search returns. The final search terms were: Mental health literacy OR depression literacy OR anxiety literacy; AND, Parent* OR Mother OR Father OR Famil* OR Caregiv*; AND, Mental health attitudes OR mental health belief* OR mental health knowledge OR mental health awareness OR stigma OR help-seeking. The full search strategy is reported in the Supplementary File ${ }^{1}$.

Ten electronic databases (PsycINFO, PsycARTICLES, SPORTDiscus, Scopus, Web of Science, CINAHL Plus, SOCindex, Humanities International Complete, MEDLINE, and Psychology and Behavioural Sciences Collection) were searched by a single researcher in May 2017 (and updated in January 2018) for eligible articles. In addition to the electronic database search, Google Scholar was searched by the same researcher using combinations of the final search terms as well as searching the key authors in the mental health literacy field (Frauenholtz, Gulliver, Jorm, Mendenhall) and the reference lists of included articles.

\section{Eligibility Criteria}

To be included in this review, studies were required to meet the following criteria: (1) be an empirical study of parent and caregiver mental health literacy as it relates to child and adolescent mental health; (2) explicitly use the phrase mental health literacy in the title, abstract, review of literature or discussion of results;

\footnotetext{
${ }^{1}$ Supplementary files documenting the search strategy, search returns and quality appraisal of individual studies are available from the authors upon request.
} 
and (3) be a peer reviewed journal article published in the English language. Studies were excluded if they did not refer explicitly to parent or caregiver mental health literacy, for example, studies examining the mental health literacy of the general public. Studies were also excluded if they did not examine parent and caregiver mental health literacy in the context of child and adolescent mental health, for example, studies examining mental health literacy of carers of the elderly.

\section{Quality Assessment}

The methodological quality of included studies was assessed by a single researcher using the Mixed Methods Appraisal Tool (MMAT; Pluye et al., 2009). The MMAT is a reliable method of assessing study quality relative to the methodology and design employed with substantial inter-rater reliability (Pace et al., 2012). Quantitative studies are sub-divided into randomised controlled trials, non-randomised trials and descriptive studies. Articles are assessed against four dimensions of quality based on design and are allocated one point per criteria met. Studies are determined to be of low methodological quality if $0-25 \%$ of criteria are met, of moderate methodological quality if 50\%-75\% of criteria are met and of high quality if $100 \%$ of criteria are met. Assessment of study quality can be found in the Supplementary File.

\section{Data Extraction and Synthesis}

The first author extracted data from all eligible studies. These data included author details, the country where the study was conducted, study design, measures used, sample size, sample characteristics and main findings (see Table 1). For quantitative studies, mental health literacy related measures and primary outcomes were extracted. For qualitative studies, data collection methods, and individual study authors' interpretations of participant quotes in the form of descriptive themes and categories were extracted. To enable integration and analysis of evidence with significant methodological heterogeneity, a narrative synthesis was undertaken (Popay et al., 2006). A narrative synthesis involves using text to summarise and explain findings (Popay et al., 2006), and can be used to organise and highlight similarities, differences or patterns across study designs, samples and findings (Centre for Review and Dissemination, 2009; Ryan, 2013).

Popay et al. (2006) outline four main elements of the narrative review process. The first element is developing a framework or theory to organise and understand findings. In this review, the mental health literacy framework as defined by Jorm et al. (1997) was used. The second element is to develop a preliminary synthesis (i.e. extracting, organising and describing findings from the studies). Quantitative and qualitative data were considered separately before merging the data (Popay et al., 2006). For qualitative studies, thematic categories as interpreted by the study authors' were extracted. For quantitative studies, outcomes and associations relevant 
to mental health literacy were interpreted and summarised textually. A thematic summary was chosen as the most appropriate method of synthesis and involved deductively categorising both quantitative and qualitative studies using the conceptual framework of the review (Thomas, Harden, \& Newman, 2012). As such, findings were categorised into the following components of mental health literacy: 1) knowledge and understanding; 2) attitudes and beliefs; 3) help-seeking categories; and, 4) mental health literacy interventions. Within these components, data were analysed inductively to facilitate the emergence of descriptive sub-themes. For example, findings first deductively coded within the "knowledge and understanding" category were inductively coded into: 1) the degree and nature of knowledge; 2) sources of knowledge, and; 3) correlates of knowledge subthemes. The third element of the narrative review is to explore the relationships in the data to investigate differences between studies. This involved examining the breadth and strength of evidence for findings within each sub-theme across studies, and the factors (e.g., sample size, measurement validity) which may explain any variation in findings. The fourth element is to examine the robustness of the synthesis based on the strength of the evidence and the ability to draw and generalise conclusions. This was achieved by a thorough methodological critique of included studies and the comparison of findings in relation to previous research.

\section{Ethical Standards and Conflict of Interest}

IRB approval was not necessary because this was a review of the literature that entailed no human subject participation. The authors report no conflict of interest.

\section{Search Returns}

The electronic databases returned 425 records and another 37 were identified through other sources.

After duplicates were removed, 296 articles were screened by title and abstract against exclusion criteria by two researchers. If uncertainty remained as to an article's eligibility, the full text of the article was scrutinised $(n=$ 97). Discrepancies were discussed before reaching a mutual agreement. The most common reasons for study exclusion were no assessment of mental health literacy $(n=42)$ or a non-parent or caregiver population $(n=30)$. In total, 20 studies were identified that met inclusion criteria. A second search was conducted in January 2018 by the lead researcher using the same search criteria but restricting the study date range to the years 2017-2018.

147 One qualitative study was identified that met inclusion criteria resulting in a total of 21 studies (see Figure 1). In addition, prominent authors as identified previously were contacted and asked to review the list of included studies and identify any missing studies. No further studies were added following this process. 


\section{Characteristics of Included Studies}

The 21 studies (see Table 1) included a total sample of 9019 parents, foster carers and other caregivers. The sample was predominantly female $(70.7 \%)$. There were 16 independent samples, with five studies (Jorm \& Wright, 2007; Jorm et al., 2007a; Jorm et al., 2007b; Jorm et al., 2008; Yap \& Jorm, 2012) using the same survey data to investigate different components of mental health literacy. Thirteen studies were quantitative, seven were qualitative, and one was mixed methods. Three studies included parent or caregivers of children and adolescents without current mental health disorders, 11 had mixed samples of children and adolescents with or without diagnosed mental health disorders, and seven had samples of children with mental health disorders/in treatment services. Thirteen studies had parent samples, four studies had foster carers or caregivers, three studies used mixed samples, and one study involved clinician perceptions about parent mental health literacy. Nine studies were conducted in Australia, six in the United States, four in the United Kingdom, one in Canada, and one in Malaysia. The quality of studies as assessed by the MMAT (Pluye et al., 2009) ranged from $25 \%$ of criteria met (two studies of low quality) to $100 \%$ of criteria met (two studies of high quality), with the majority of studies assessed as moderate quality Qualitative studies were primarily limited by the lack of evidence of researcher reflexivity, whereas quantitative studies were limited by restrictive sampling strategies, inadequate sample representativeness, and the use of non-validated measures. Results are presented within the three major categories of mental health literacy: (a) knowledge and understanding; (b) attitudes and beliefs; (c) helpseeking; and, a fourth category of (d) mental health literacy interventions (see Table 2 Supplementary File).

\section{Knowledge and Understanding}

Seventeen studies investigated parents and caregivers' knowledge or understanding of mental health issues and help-seeking. Various dimensions of mental health knowledge were measured including problem or symptom recognition, the amount, accuracy and source of knowledge, mental health awareness, and knowledge of depression, anxiety mood disorders, and treatment options. This section is divided into three sub-themes representing: (a) degree and nature of knowledge; (b) sources of knowledge; and, (c) correlates of knowledge.

Degree and nature of knowledge. There were mixed findings relating to mental health knowledge of parents and caregivers with studies highlighting strengths and gaps in knowledge. Five cross-sectional studies examined parents' recognition of mental health disorders symptoms (Bonfield, Guishard-Pine, \& Langdon, 2010; Frauenholtz, Conrad Hiebner, \& Mendenhall, 2015; Lawrence et al., 2015; Mendenhall \& Frauenholtz, 2015; Shah et al., 2004). In one study, mothers had high problem recognition accuracy with $91.0 \%$ correctly identifying a mental health problem from a depression vignette, and $84.0 \%$ from an anxiety vignette (Shah et al., 
2004). Similarly, $86.0 \%$ of foster carers were able to accurately recognise a mental health problem from a vignette (Bonfield, Guishard-Pine, \& Langdon, 2010) but this was not associated with their ability to detect a mental health problem in their child. In another study, $66.7 \%$ of mental health providers indicated that parents' mental health knowledge was strongest in terms of identifying symptoms (Frauenholtz, Conrad Hiebner, \& Mendenhall, 2015). However, in the same study almost half (44.8\%) reported that parents had very little or no knowledge of youth mental health disorders overall, with $79.3 \%$ rating parent knowledge as limited, mostly inaccurate and inconsistent, especially in terms of prevention, treatment and help-seeking options. In another cross-sectional study, parents of youths with mood disorders were found to have some limited knowledge of mood disorders and treatment (Mendenhall \& Frauenholtz, 2015). Just over a quarter of Australian parents $(27.3 \%)$ reported being uncertain if their child or adolescent needed help for a mental health disorder, with two in five parents unsure of where to seek help (Lawrence et al., 2015).

Five qualitative studies investigated parent and caregiver mental health knowledge. Two studies revealed that parents of young athletes (Hurley et al., 2017), and immigrant mothers (Montgomery \& Terrion, 2016) had some knowledge and understanding of supportive actions and prevention strategies such as engaging in discussions about mental health with their adolescents. However, parents of young athletes felt they had inadequate knowledge of mental health disorder symptoms, how to handle mental health disorders, or how to communicate about mental health (Hurley et al., 2017). There is some indication that the level of parent and caregiver knowledge differs across cultural groups. Malaysian caregivers were found to have limited knowledge of mental illness and treatment, associated with religious and spiritual beliefs of unknown and supernatural causes of mental illness (Mohammad et al., 2012). Korean-American parents had limited knowledge and some misconceptions about the causes of depression and its treatment (Jeong, McCreary, \& Hughes, 2017). The study also found that Korean fathers had less knowledge about depressive symptoms and treatment than mothers with their primary focus on earning and providing for the household (Jeong et al., 2017).

Sources of knowledge. Parent and caregivers identified both formal and informal sources of mental health information. In three qualitative studies, those parents and caregivers with experience of mental health disorders reported that they learned about mental health and treatment options through contact with mental health professionals (Honey et al., 2015; Mohammad et al., 2012; Umpierre et al., 2015). Caregivers in Malaysia described learning through the experience of caring for a person with mental illness (Mohammad et al., 2012). Latino parents and caregivers indicated that they had little knowledge about child mental health services until they entered into care (Umpierre et al., 2015). Friends and co-workers were identified by $70.1 \%$ of mental health 
210 professionals as the primary source of parents' knowledge, with family the second biggest influence

211 (Frauenholtz et al., 2015). Parents and caregivers reported that they gain information and advice from friends,

212 family and peers (Honey et al., 2015, Umpierre et al., 2015; York \& Jones, 2017) and learned about mental

213 health through exposure to online and print material (Frauenholtz, Conrad-Hiebner, \& Mendenhall, 2015;

214 Honey et al., 2015; Mohammad et al., 2012).

215 Correlates of knowledge. Personal and family experience of mental health issues and help-seeking, as well as exposure to mental health related information, were associated with greater parent and caregiver mental health knowledge in five studies (Frauenholtz, et al., 2015; Honey et al., 2015; Hurley et al., 2017; Lopez et al., 2009; Mendenhall \& Frauenholtz, 2015). Diagnosis of a family member (77.0\%) and prior treatment experiences $(75.9 \%)$ were identified by mental health professionals as the primary influences on parent and caregiver knowledge (Frauenholtz et al., 2015). Exposure to mental health information, but not parent mental health history, significantly correlated with Australian parents’ prevention knowledge (Yap \& Jorm, 2012). One study found that child and parent disorder diagnosis, and current use of treatment services were associated with greater knowledge of mood disorders (Mendenhall \& Frauenholtz, 2015). That study also found that child bipolar diagnosis and current in-family use of mental health services were associated with knowledge of treatment services. Other correlates of mental health knowledge included being highly educated, female, Caucasian, having older children, and having a higher perceived need for treatment (Mendenhall \& Frauenholtz, 2015). Parents' level of education was also reported by $75.9 \%$ of mental health professionals as an influence on parent mental health knowledge, but child and parent sex, age and race were not considered influential (Frauenholtz et al., 2015).

\section{Attitudes and Beliefs}

Sixteen studies explored parent and caregiver attitudes and beliefs about mental health and help seeking. The following subthemes were represented: (a) stigma; (b) role of parent; (c) attitudes to help-seeking; and, (d) fear and worry.

Stigma. The relationships between stigma, knowledge, and help-seeking were mixed, as reported in five qualitative studies (Honey et al., 2015; Hurley et al., 2017; Montgomery \& Terrion, 2016; Mohammad et al., 2012; Umpierre et al., 2015). Immigrant mothers espoused stigmatising attitudes about mental illness despite having knowledge of mental health and help-seeking options (Montgomery \& Terrion, 2016). Conversely, parents of youth athletes perceived their mental health literacy as limited, but the majority were open to mental health discussion and education and wanted to reduce the public stigma attached to mental illness and help- 
seeking (Hurley et al., 2017). Three studies reported that some parents and caregivers were fearful, mistrustful and reluctant in accessing services until their child became unwell and they entered into care (Honey et al., 2015; Mohammad et al., 2012; Umpierre et al., 2015). Parents and caregivers in these studies originally believed that others in their community networks would view mental illness and help-seeking negatively but their attitudes became more positive through contact with mental health professionals and gaining more knowledge.

Role of parent in youth mental health. Qualitative findings from four studies revealed that the majority of parents and caregivers perceived that they had a role and responsibility in supporting youth mental health but there were differences in how confident they felt about this role. Canadian immigrant mothers felt positive about their role and were confident in their ability to use their experience and knowledge to support their child's mental health (Montgomery \& Terrion, 2016). Parents of young male athletes also recognised their role but were not confident in their ability to communicate and actively support and protect their children's mental health (Hurley et al., 2017). Parents of adolescents with mental health disorders were proactive in seeking knowledge and maximising support resources available to them (Honey et al., 2015). Korean-American parents perceived that parenting style and family issues were one of the main causes of depression and that changing parenting style could be effective in treating depression (Jeong et al., 2017). A sample of Australian parents believed that their behaviour could aid prevention of mental health disorders (Yap \& Jorm, 2012). For example, $92.0 \%$ of parents perceived that "showing the child lots of affection" can protect against the development of mental health disorders.

Attitudes to help-seeking. Parents and caregivers believed in the value and importance of seeking help and using services in six studies (Bonfield, Guishard-Pine, \& Langdon, 2010; Honey et al., 2015; Hurley et al., 2017; 2018; Mohammad et al., 2012; Montgomery \& Terrion, 2016). Parent and caregivers in four qualitative studies reported that they had taken or were willing to take whatever steps necessary to help and support their child through a mental health problem. A cross-sectional study found that foster carers' favourable attitudes to help-seeking were associated with both contacting and referral to mental health services but not actual service use (Bonfield et al., 2010). Previous exposure and experience of mental illness and service use was associated with a willingness to seek help in two qualitative studies (Honey et al., 2015; Hurley et al., 2017). In contrast, parents in two other qualitative studies held negative attitudes (e.g. fear, mistrust, lack of faith) to mental health services that was based on observation of others' unsuccessful help-seeking experience (Jeong et al., 2017; Umpierre et al., 2015). 
Fear and worry. Three focus group studies revealed that parents expressed concerned about child mental health and help-seeking (Hurley et al., 2017; Montgomery \& Terrion, 2016; Umpierre et al., 2015). Parents of youth athletes were fearful of the potential impact of mental health disorders on their child and their lack of knowledge about how to help (Hurley et al., 2017). Immigrant mothers were also worried about giving mental health information to children fearing that such knowledge might evoke a negative, undesirable reaction (Montgomery \& Terrion, 2016). Fear and mistrust were also barriers to seeking help, with Latino parents referring to negative mental health treatment stories and the belief that children could be taken away by social services (Umpierre et al., 2015).

\section{Help-seeking and Support}

This category encapsulates how parents and caregivers access help and support and what action strategies they put in place to support and protect youth mental health. The section is divided into three subthemes: (a) sources of support; (b) help-seeking strategies; and, (c) factors influencing help-seeking.

Sources of support. Formal and informal sources of support played an important role in parents' knowledge, beliefs and practices about mental health and help seeking. Australian parents in cross-sectional research samples endorsed speaking to family and friends as an appropriate help-seeking response (Jorm \& Wright, 2007; Jorm et al., 2007b; Jorm et al., 2008). For example, 86.0\% of parents of 12-17 year olds believed it helpful to engage the support of close family, and $82.0 \%$ believed in the helpfulness of close friends (Jorm \& Wright, 2007). In addition, mental health providers rated informal sources of support, such as family and friends, as parents' primary source of information on mental health issues (Frauenholtz et al., 2015). Four qualitative studies revealed that parents and caregivers had positive views about, and sought support and advice from informal sources such as friends, family, peers and co-workers (Honey et al., 2015; Hurley et al., 2017; Umpierre et al., 2015; York \& Jones, 2017). In particular, Latino parents identified close female relatives such as mothers and sisters as particularly valuable sources of support (Umpierre et al., 2015). Parents of youth sport athletes emphasised the importance of close family and community support networks (Hurley et al., 2017), and Malaysian caregivers valued traditional healers in the community (Mohammad et al., 2012). professional help (65.0\%; Jorm et al., 2007b) or would suggest to seek professional help (73.3\%; Jorm et al., 2008) when asked to rank response strategies to a youth mental health issue. Open ended responses also revealed parents' intentions to seek help from a mental health specialist or service (Jorm et al., 2007a). General practitioners were endorsed by Australian parents as their preferred first option for seeking professional help and 
299

300

301

302

303

304

305

306

307

308

309

310

311

312

313

314

315

316

317

318

319

320

321

322

323

324

325

326

327

328

advice in five cross-sectional studies, with endorsement ranging from $55.0 \%$ (Jorm et al., 2007b) to $95.0 \%$

(Jorm \& Wright, 2007) of parents. Counsellors were rated more favourably than mental health specialists such as psychologists and psychiatrists (Jorm et al., 2007a; Jorm et al., 2007b), and psychologists were rated more favourably than psychiatrists (Jorm \& Wright, 2007; Jorm et al., 2007b).

Parents in Australian samples rated medication, hospitalisation, dealing with the problem alone, and talking about suicide as negative or harmful (Jorm \& Wright, 2007; Jorm et al., 2007b; Jorm, et al., 2008). Parents endorsed listening, talking and supporting as an appropriate first course of action (Jorm et al., 2008; Jorm, et al., 2007b). In addition, parents rated self-help intervention strategies, such as physical activity (91.0\%) and relaxation training (93.0\%), as helpful (Jorm \& Wright, 2007; Jorm et al., 2008). Counselling was rated positively in two studies (Jorm \& Wright, 2007; Jorm et al., 2007a), with 44.0\% of Australian parents and caregivers identifying counselling on how to manage a mental health problem as their biggest perceived need for help (Lawrence et al., 2015). In contrast, counselling was perceived as mostly ineffective by KoreanAmerican parents due to perceived lack of improvement and slow progress (Jeong et al., 2017).

Factors influencing help-seeking. Ethnic, cultural and religious affiliation were related to parents' perceptions of appropriate help-seeking strategies (Honey et al., 2015; Jeong et al., 2017; Mohammad et al., 2012; Shah et al., 2004). For example, fewer Pakistani mothers (64\%) would seek professional help for psychosis compared to Caucasian (88\%) mothers (Shah et al., 2004). For caregivers in Malaysia, mental health services were limited, and caregivers tried alternative treatments, based on religious or spiritual beliefs (Mohammad et al., 2012). Korean-American fathers had the final say in seeking help for their child as they were the primary income earners and thus the decision maker in Korean culture (Jeong et al., 2017).

The nature of the mental health problem was also an important factor in whether or not parents would take action and seek help. For example, qualitative studies indicated that parents and caregivers were likely to take action if they observed children's disruptive externalising behaviour or changes in personality (Honey et al., 2015; Hurley et al., 2017; Jeong et al., 2017; York \& Jones, 2017). In a cross-sectional study, the presence of a mental health problem in a child (scoring in the abnormal or borderline range) was associated with a greater likelihood of problem detection, referral to mental health services, and service use (Bonfield et al., 2010). In another study with Australian parents, the greater impact of a mental health disorder on youth daily functioning was associated with a greater perceived need for parental help, with $100 \%$ of parents of youths with a severe mental health disorder perceiving need for help compared to $68.3 \%$ of parents of youths with a mild mental health disorder (Lawrence et al., 2015). 
The most prominent barriers to help-seeking across studies were financial difficulties (Hurley et al.,

2017; Jeong et al, 2017; Jorm et al., 2007a; Lawrence et al., 2015; Umpierre et al., 2015), inadequate mental health knowledge (Hurley et al., 2017; Jeong, et al., 2017; Lawrence et al., 2015; Mohammad et al., 2012), and availability of services (Honey et al., 2015; Jeong et al., 2017; Lawrence et al., 2015; Mohammad et al., 2012). Other barriers included child resistance (Jorm et al., 2007a; Lawrence et al., 2015), time (Honey et al., 2015; Hurley et al., 2017; York \& Jones, 2017), fear and suspicion of services (Montgomery \& Terrion, 2016; Umpierre et al., 2015), lack of parent confidence (Honey et al., 2015, Hurley et al., 2017), and preferring to handle the problem by themselves or with the help of family or friends (Lawrence et al., 2015).

\section{Mental Health Literacy Interventions}

There were four interventions, of which two were controlled trials and two were non-controlled trials.

The first (non-controlled) trial tested a counselling skills intervention to increase the mental health awareness and coping strategies of ten foster carers (Mosuro, Malcolm, \& Guishard-Pine, 2014). The study found that mental health awareness was higher following the intervention but no change was found in coping skills. The second (non-controlled) trial aimed to increase psychosis literacy (specifically the identification of symptoms of psychosis, and knowledge of professional help-seeking options) in Latino caregivers of children with schizophrenia and other Latino community residents (Lopez et al., 2009). The study revealed increases in knowledge of symptoms and confidence in both caregiver and resident samples. There were also increases in problem attributions to psychosis and mental illness, and a greater likelihood to recommend professional helpseeking among residents but not caregivers.

Two controlled interventions aimed to increase the mental health literacy of parents in the workplace and in community sport clubs. The first study developed four interactive modules covering anxiety, depression, treatment options and what parents can do (Deitz et al., 2009). The intervention was delivered online through parents' workplace and aimed to increase parent's knowledge and confidence in dealing with mental health issues. It was found that parents receiving the intervention had greater knowledge of youth mental health issues and greater self-efficacy in handling these issues compared to a waitlist control group. No significant differences were found between those parents with known or suspected mental health problems and those without. adolescents through community sport clubs (Hurley et al., 2018). A face-to-face workshop covered symptoms of depression and anxiety, communicating about mental health, and mental health resources and treatment options. 
359

360

361

362

363

364

365

366

367

368

369

370

371

372

373

374

375

376

377

378

379

380

381

382

383

384

385

386

387

388

options, and confidence to assist someone experiencing a mental health disorder, compared to parents in a matched sport club community control group. Qualitative evaluation of the intervention revealed that parent's awareness had increased, they had applied knowledge from the intervention and used it as a catalyst for discussion of mental health in their families.

\section{Discussion}

This systematic review aimed to synthesise and evaluate research on parent and caregiver mental health literacy referent to youth mental health. There were mixed findings relating to parent and caregiver knowledge of mental health and help-seeking options. Previous experience of mental health disorders and help-seeking was associated with greater knowledge but the relationship between past experience and help-seeking attitudes was less clear. Overall, parents and caregivers were in favour of seeking help with some exceptions based on fear, mistrust, and stigma. Parents and caregivers used both formal and informal sources of support, with general practitioners the preferred professional help-seeking option. Multiple factors influenced parent and caregivers' ability to support and help young people with cultural and religious beliefs, financial and knowledge barriers, and the severity and impact of the mental health problem the most prominent factors. Four interventions reported some increases in mental health knowledge. Findings should be interpreted with caution due to limitations of the evidence base such as restrictive sampling strategies, non-representative samples, inconsistent and non-validated measures, and lack of longitudinal and controlled trial studies.

Variation in the findings relating to parent and caregiver knowledge may be due to the different types of knowledge measured such as symptom identification, prevention and self-help strategies, and knowledge of treatment and help-seeking options, as well as different knowledge measurement tools. Previous personal and familial experience of mental health disorders and help-seeking as well as experience in the mental health caregiver role was associated with greater mental health knowledge in the majority of studies, supporting previous research (Jung, Von Sternberg, \& Davis, 2017; Mendenhall \& Frauenholtz, 2013). This may be due to parent and caregiver interaction with mental health professionals, a prominent source of information and advice as evidenced in this review. However, a lack of prevention studies with parent or caregiver samples prohibits comparison of knowledge between those in treatment and those not. The nature of the relationship and interaction between different sociodemographic variables such as age, education and gender on parent and caregiver knowledge remains unclear with sample diversity an issue. Indeed, a recent large scale study of over 16,500 Australians found no clear evidence of age or gender as predictive of mental health literacy and found only mixed associations for participants' exposure to mental illness (Cutler, Reavley, \& Jorm, 2017). 
Previous personal experience of help-seeking was associated with increased willingness to seek help.

This review also found that once in care, parents' negative attitudes appeared to diminish as they gained more

391

392

393

394

395

396

397

398

399

400

401

402

403

404

405

406

407

408

409

410

411

412

413

414

415

416

417

418

knowledge, indicating the relationship between knowledge, attitudes and help-seeking components of mental

health literacy. Research has indeed shown that help-seeking experience among parents and caregivers has been associated with more positive help-seeking attitudes, reduced stigmatisation, greater help-seeking intentions, , and higher use of services in adolescents (Sherman \& Ali, 2017; Turner \& Liew, 2010). There was a preference for parents and caregivers, regardless of mental health status, to seek and use help from informal sources of support in line with previous research (Gulliver et al., 2012; Reardon et al., 2017). More positive attitudes to help-seeking are associated with support and encouragement from within social networks such as family and friends (Jung, Von Sternberg, \& Davis, 2017). However, in this review exposure to others' negative helpseeking experiences and attitudes was associated with more negative parent attitudes. Thus, social networks have the potential to influence parent and caregiver attitudes and inhibit or facilitate help-seeking practices.

\section{Methodological Critique of Included Studies}

The majority of the quantitative studies included were cross sectional with an absence of longitudinal research limiting the ability to infer causality. In addition, four cross-sectional studies simply reported the percentage frequency of responses of parents and caregivers endorsing various help-seeking sources and services. These studies did not account for previous mental health experience or knowledge and predominantly involved parents rating behaviours as recommended by the public and mental health first aid manuals. However, closed questions or options may yield different responses than open ended options and may not reflect what parents or caregivers would actually do to help their child (Angermeyer, 2017).

In this review, there was no universal or consistent measure of mental health literacy employed. There was no evidence provided for the psychometric validity of measures in 9/15 (60\%) studies. Eleven studies developed some or all of their own study measures while hypothetical vignettes were used in 8/15 (53.3\%) quantitative studies. Furthermore, these vignettes were not consistent - varying in terms of type and number of disorders presented and questions or statements following. This can be problematic as knowledge may differ based on disorder, with previous research showing greatest public knowledge of depression and substance abuse and lowest for anxiety and psychosis (Cutler, Reavley, \& Jorm, 2017). Vignettes may be limited in their ability to distinguish between different components of mental health literacy, and cannot provide total or sub-scale (e.g., knowledge, attitudes) scores of mental health literacy (O’Connor \& Casey, 2014). Furthermore, participants may not respond in the same way to a hypothetical vignette as they would to their own child. 
volunteered may have had more knowledge and positive attitudes towards mental health and help-seeking. al., 2008; Teagle, 2002). Only one study investigated the views of mental health professionals on parents' mental health literacy (Frauenholtz et al., 2015). Considering the potential bias of self-selection, more otherreport studies from key individuals in the youth mental health sphere would be beneficial. However, such an approach would need to acknowledge potential limitations in others' perceptions, such as inaccuracy, bias and the nature of their relationship and experience with parents.

\section{Limitations}

Findings are limited and should be interpreted with caution in light of the methodological limitations of the review. As a narrative review was conducted to amalgamate research findings from different study designs, it was not possible to statistically pool quantitative findings or determine the strength of associations between knowledge, attitudes, help-seeking behaviours and other key variables. Additionally, findings investigating parent and caregiver attitudes and beliefs are limited by a lack of experimental research.

Almost all studies that met inclusion criteria were conducted in Western, developed countries. et al., 2012). In addition, most studies were conducted in Australia where the term mental health literacy was coined and large scale public awareness campaigns have been conducted revealing significant improvements in the public's mental health literacy (Jorm, Christensen, \& Griffiths, 2006; Reavley \& Jorm, 2011). A recent review highlighted that mental health literacy in non-western countries is poorly understood (Furnham \& Hamid, 2014). Indeed, there is a lack of studies in non-western countries which may have different religious or cultural belief and value systems, as well as limited access to mental health education and treatment services.

\section{Research Implications}

The limitations discussed above yield several implications for research at the individual study and review level. Despite mental health literacy accounting for knowledge, attitudes and help-seeking practices, multiple studies in the current review applied the term mental health literacy solely in describing knowledge outcomes. We contend that researchers within this field should endeavour to use the term mental health literacy to account for the relationship between knowledge, attitudes and help-seeking as these variables are interrelated and findings may be limited when examined in isolation. As such, future research should examine how these key components interrelate with each other and other key variables. For example, the association between 
mental health literacy and relevant socio-demographic variables is not clear and needs to be consistently tested across diverse samples. Longitudinal research also needs to be employed to examine the stability of parent and caregiver mental health literacy over time.

Future studies should aim to employ different sampling strategies to reach, engage and robustly test findings with more representative samples of parents and caregivers with a particular focus on fathers, other male caregivers, and non-Western populations. Further studies need to be conducted on parents and caregivers without any experience of mental health issues and examine their mental health literacy in a preventative and promotion context. Future research also needs to employ a reliable and valid measure of parent and caregiver mental health literacy. Research is warranted on the potential positive or harmful effects of increasing mental health literacy on one's own psychological distress and well-being. This relationship is still unclear with studies in the general public reporting both negative (e.g., Goldney, Eckert, Hawthorne, \& Taylor, 2010) and positive (e.g., Brijnath et al. 2016) associations. Studies should also investigate the direct or indirect effect of increased parent and caregiver mental health literacy on child and adolescent psychological and treatment outcomes.

\section{Implications for Mental Health Literacy Education Programmes}

Despite recent improvements at a population level, parent and caregiver mental health literacy remains inadequate. There is an important need for targeted mental health education programmes to aid in the prevention, management and treatment of youth mental health. Intervention studies in this review yielded mixed results with some significant increases in parent and caregiver mental health knowledge and confidence to assist someone experiencing mental health symptoms, in line with previous review findings (Morgan, Ross, \& Reavley, 2018). However, it is still unclear whether these interventions influenced actual help-seeking behaviours for participants themselves or towards others. As such, interventions need to include mechanisms to determine the transfer of benefit over time from participants to others (Anderson \& Pierce, 2012).

Findings from this review suggest that parents and caregivers appear receptive to receiving mental health related information and recognise the importance of their role in youth mental health. However, interventions need to be made accessible to parents and take into account their preferences for content and delivery. For example, brief interventions and online interventions can overcome reported time and cost barriers and be effective in raising awareness and knowledge, but may be limited in their capacity to change long- or culturally-held attitudes to mental health and help-seeking. Intervention content and delivery should also be adapted to the target audience. For example, two studies in this review developed intervention messages and content, and used tailored delivery methods to match the preferences of Latino parents (Lopez et al., 2009; 
479 Umpierre et al., 2015). Two further studies delivered intervention content in novel settings to maximise

480 convenience and accessibility by reaching parents where they already are, such as in community sport clubs

481 (Hurley et al., 2018) and in the workplace (Deitz et al., 2009). Content and delivery also needs to be adapted to

482 the mental health status and experience of participants, e.g. in a prevention, help-seeking or already in-treatment

483 context. For example, parenting programs (Sanders et al., 2014), and health activation interventions (Hibbard \&

484 Gilbert, 2014) have adapted intervention content, intensity and delivery to match the identified level and needs

485 of participants. In addition, this review suggests the importance of engaging parent networks in education

486 programs to increase reach and impact within the community. Indeed, peer support networks are proposed as

487 catalytic in improving health outcomes in parents (Keller \& McDade, 2000), and expanding mental health

488 training for those in a position of responsibility or trust can influence help-seeking in others (Jung, Von

489 Sternberg, \& Davis, 2017). To test the effectiveness of any future education and intervention programmes more

490 randomised controlled trials with larger, more diverse samples are needed.

491

492

493

494

495

496

497

498

499

500

501

502

503

504

505

506

507

508 


\section{References}

*Included in systematic review

Alegría, M., Polo, A., Gao, S., Santana, L., Rothstein, D., Jimenez, A., ... \& Normand, S. L. (2008). Evaluation of a patient activation and empowerment intervention in mental health care. Medical Care, 46, 247 256.

Anderson, R. J., \& Pierce, D. (2012). Assumptions associated with mental health literacy training-Insights from initiatives in rural Australia. Advances in Mental Health, 10, 258-267.

*Bonfield, S., Collins, S., Guishard-Pine, J., \& Langdon, P. E. (2009). Help-seeking by foster-carers for their looked after children: the role of mental health literacy and treatment attitudes. British Journal of Social Work, 40, 1335-1352.

Brijnath, B., Protheroe, J., Mahtani, K. R., \& Antoniades, J. (2016). Do web-based mental health literacy interventions improve the mental health literacy of adult consumers? Results from a systematic review. Journal of Medical Internet Research, 18(6), e165. http://doi.org/10.2196/jmir.5463

Centre for Reviews and Dissemination (2009). Systematic Reviews: CRD's guidance for undertaking reviews in health care. York: University of York.

Cutler, T. L., Reavley, N. J., \& Jorm, A. F. (2017). How 'mental health smart'are you? Analysis of responses to an Australian Broadcasting Corporation News website quiz. Advances in Mental Health. Advance online publication. doi:10.1080/18387357.2017.1317581

*Deitz, D. K., Cook, R. F., Billings, D. W., \& Hendrickson, A. (2009). Brief report: a web-based mental health program: reaching parents at work. Journal of Paediatric Psychology, 35, 488-494.

*Frauenholtz, S., Conrad-Hiebner, A., \& Mendenhall, A. N. (2015). Children's mental health providers' perceptions of mental health literacy among parents and caregivers. Journal of Family Social Work, 18, 40-56.

Furnham, A., \& Hamid, A. (2014). Mental health literacy in non-western countries: a review of the recent literature. Mental Health Review Journal, 19, 84-98.

Goldney, R. D., Eckert, K. A., Hawthorne, G., \& Taylor, A. W. (2010). Changes in the prevalence of major depression in an Australian community sample between 1998 and 2008. Australian \& New Zealand Journal of Psychiatry, 44(10), 901-910. 
538

539

540

541

542

543

544

545

546

547

548

549

550

551

552

553

554

555

556

557

558

559

560

561

562

563

564

565

566

Greene, J., \& Hibbard, J. H. (2012). Why does patient activation matter? An examination of the relationships between patient activation and health-related outcomes. Journal of General Internal Medicine, 27, 520526.

Green, C. A., Perrin, N. A., Polen, M. R., Leo, M. C., Hibbard, J. H., \& Tusler, M. (2010). Development of the patient activation measure for mental health. Administration and Policy in Mental Health and Mental Health Services Research, 37, 327-333.

Gronholm, P. C., Ford, T., Roberts, R. E., Thornicroft, G., Laurens, K. R., \& Evans-Lacko, S. (2015). Mental health service use by young people: the role of caregiver characteristics. PloS One, 10(3), e0120004.

Gulliver, A., Griffiths, K. M., \& Christensen, H. (2010). Perceived barriers and facilitators to mental health help-seeking in young people: a systematic review. BMC Psychiatry, 10, 113-122. doi: 10.1186/1471$244 \mathrm{X}-10-113$

Gulliver, A., Griffiths, K. M., Christensen, H., \& Brewer, J. L. (2012). A systematic review of help-seeking interventions for depression, anxiety and general psychological distress. BMC Psychiatry, 12, 81-92. doi:10.1186/1471-244X-12-81

Hibbard, J. (2017). Patient activation and health literacy: what's the difference? How do each contribute to health outcomes. Study of Health Technology and Informatics, 240, 251-262.

Hibbard, J., \& Gilburt, H. (2014). Supporting people to manage their health. An introduction to patient activation. London, UK: The King's Fund.

Honey, A., Alchin, S., \& Hancock, N. (2014). Promoting mental health and wellbeing for a young person with a mental illness: parent occupations. Australian Occupational Therapy Journal, 61, 194-203.

*Honey, A., Chesterman, S., Hancock, N., Llewellyn, G., Hazell, P., \& Clarke, S. (2015). Knowing what to do and being able to do it: Influences on parent choice and use of practices to support young people living with mental illness. Community Mental Health Journal, 51, 841-851.

*Hurley, D., Swann, C., Allen, M. S., Okely, A. D., \& Vella, S. A. (2017). The role of community sports clubs in adolescent mental health: the perspectives of adolescent males' parents. Qualitative Research in Sport, Exercise and Health, 9, 372-388.

*Hurley, D., Allen, M. S., Swann, C., Okely, A. D., \& Vella, S. A. (2018). The development, pilot, and process evaluation of a parent mental health literacy intervention through community sports clubs. Journal of Child and Family Studies, 1-12. 
567

568

569

570

571

572

573

574

575

576

577

578

579

580

581

582

583

584

585

586

587

588

589

590

591

592

593

594

595

*Jeong, Y. M., McCreary, L. L., \& Hughes, T. L. (2017). Qualitative study of depression literacy among Korean American parents of adolescents. Journal of Psychosocial Nursing and Mental Health Services, 56, 4856.

Jorm, A. F., Christensen, H., \& Griffiths, K. M. (2006). Changes in depression awareness and attitudes in Australia: the impact of beyondblue: the national depression initiative. Australian \& New Zealand Journal of Psychiatry, 40, 42-46.

Jorm, A. F., Korten, A. E., Jacomb, P. A., Christensen, H., Rodgers, B., \& Pollitt, P. (1997). Mental health literacy: A survey of the public's ability to recognise mental disorders and their beliefs about the effectiveness of treatment. Medical Journal of Australia, 166, 182-186.

*Jorm, A. F., Wright, A., \& Morgan, A. J. (2007a). Where to seek help for a mental disorder? The Medical Journal of Australia, 187, 556-560.

*Jorm, A. F., Wright, A., \& Morgan, A. J. (2007b). Beliefs about appropriate first aid for young people with mental disorders: findings from an Australian national survey of youth and parents. Early Intervention in Psychiatry, 1, 61-70.

*Jorm, A. F., \& Wright, A. (2007). Beliefs of young people and their parents about the effectiveness of interventions for mental disorders. Australian and New Zealand Journal of Psychiatry, 41, 656-666.

*Jorm, A. F., Morgan, A. J., \& Wright, A. (2008). First aid strategies that are helpful to young people developing a mental disorder: beliefs of health professionals compared to young people and parents. BMC Psychiatry, 8, 42-52.

Jorm, A. F. (2012). Mental Health Literacy: Empowering the Community to Take Action for Better Mental Health. American Psychologist, 67, 231-243.

Jung, H., von Sternberg, K., \& Davis, K. (2017). The impact of mental health literacy, stigma, and social support on attitudes toward mental health help-seeking. International Journal of Mental Health Promotion, 19, $252-267$.

Kelly, C. M., Mithen, J. M., Fischer, J. A., Kitchener, B. A., Jorm, A. F., Lowe, A., \& Scanlan, C. (2011). Youth mental health first aid: a description of the program and an initial evaluation. International Journal of Mental Health Systems, 5, 4-12.

Kieling, C., Baker-Henningham, H., Belfer, M., Conti, G., Ertem, I., Omigbodun, O., ... \& Rahman, A. (2011). Child and adolescent mental health worldwide: evidence for action. The Lancet, 378, 1515-1525. 
596

597

598

599

600

601

602

603

604

605

606

607

608

609

610

611

612

613

614

615

616

617

618

619

620

621

622

623

Kitchener, B. A., \& Jorm, A. F. (2002). Mental health first aid training for the public: evaluation of effects on knowledge, attitudes and helping behaviour. BMC Psychiatry, 2, 10-15.

*Lawrence, D., Johnson, S., Hafekost, J., Boterhoven De Haan, K., Sawyer, M., Ainley, J., \& Zubrick, S. R. (2015). The Mental Health of Children and Adolescents. Report on the second Australian Child and Adolescent Survey of Mental Health and Wellbeing. Canberra: Department of Health

*López, S. R., Lara, M. D. C., Kopelowicz, A., Solano, S., Foncerrada, H., \& Aguilera, A. (2009). La Clave to increase psychosis literacy of Spanish-speaking community residents and family caregivers. Journal of Consulting and Clinical Psychology, 77, 763-774.

Mendenhall, A. (2012). Predictors of service utilization among youth diagnosed with mood disorders. Journal of Child \& Family Studies, 21, 603-611. doi:10.1007/s10826-011-9512-x

Mendenhall, A. N., \& Frauenholtz, S. (2013). Mental health literacy: social work's role in improving public mental health. Social Work, 58, 365-368.

*Mendenhall, A. N., \& Frauenholtz, S. (2015). Predictors of mental health literacy among parents of youth diagnosed with mood disorders. Child \& Family Social Work, 20, 300-309. doi:10.1111/cfs.12078

*Mohamad, M. S., Zabidah, P., Fauziah, I., \& Sarnon, N. (2012). Mental health literacy among family caregivers of schizophrenia patients. Asian Social Science, 8, 74-82.

Moher, D., Shamseer, L., Clarke, M., Ghersi, D., Liberati, A., Petticrew, M., ... \& Stewart, L. A. (2015). Preferred reporting items for systematic review and meta-analysis protocols (PRISMA-P) 2015 statement. Systematic Reviews, 4, 1-8.

*Montgomery, N. D., \& Lennox Terrion, J. (2016). Tensions along the path toward mental health literacy for new immigrant mothers: perspectives on mental health and mental illness. International Journal of Mental Health Promotion, 18, 83-94.

Morgan, A. J., Ross, A., \& Reavley, N. J. (2018). Systematic review and meta-analysis of Mental Health First Aid training: effects on knowledge, stigma, and helping behaviour. PloS One, 13, e0197102.

*Mosuro, S., Malcolm, D., \& Guishard-Pine, J. (2014). Mental health awareness and coping in foster carers: The impact of a counselling skills intervention. Educational and Child Psychology, 31, 64-70.

O’Connor, M., Casey, L., \& Clough, B. (2014). Measuring mental health literacy-a review of scale-based measures. Journal of Mental Health, 23, 197-204. 
624

625

626

627

628

629

630

631

632

633

634

635

636

637

638

639

640

641

642

643

644

645

646

647

648

649

650

651

652

653

Pace, R., Pluye, P., Bartlett, G., Macaulay, A. C., Salsberg, J., Jagosh, J., \& Seller, R. (2012). Testing the reliability and efficiency of the pilot Mixed Methods Appraisal Tool (MMAT) for systematic mixed studies review. International Journal of Nursing Studies, 49, 47-53.

Pluye, P., Gagnon, M. P., Griffiths, F., \& Johnson-Lafleur, J. (2009). A scoring system for appraising mixed methods research, and concomitantly appraising qualitative, quantitative and mixed methods primary studies in mixed studies reviews. International Journal of Nursing Studies, 46, 529-546.

Polanczyk, G. V., Salum, G. A., Sugaya, L. S., Caye, A., \& Rohde, L. A. (2015). Annual Research Review: A meta-analysis of the worldwide prevalence of mental disorders in children and adolescents. Journal of Child Psychology and Psychiatry, 56, 345-365.

Popay, J., Roberts, H., Sowden, A., Petticrew, M., Arai, L., Rodgers, M., ... \& Duffy, S. (2006). Guidance on the conduct of narrative synthesis in systematic reviews. A product from the ESRC methods programme. UK: University of Lancaster.

Reardon, T., Harvey, K., Baranowska, M., O’Brien, D., Smith, L., \& Creswell, C. (2017). What do parents perceive are the barriers and facilitators to accessing psychological treatment for mental health problems in children and adolescents? A systematic review of qualitative and quantitative studies. European Child \& Adolescent Psychiatry, 26, 623-647

Reavley, N. J., \& Jorm, A. F. (2011). Recognition of mental disorders and beliefs about treatment and outcome: findings from an Australian national survey of mental health literacy and stigma. Australian \& New Zealand Journal of Psychiatry, 45, 947-956.

Reavley, N. J., \& Jorm A. F. (2012). Young people's stigmatizing attitudes towards people with mental disorders: findings from an Australian national survey. Australian and New Zealand Journal of Psychiatry, 45, 1033-1039.

Rickwood, D. J., Deane, F. P., \& Wilson, C. J. (2007). When and how do young people seek professional help for mental health problems. Medical Journal of Australia, 187, S35-S39.

Ryan, R. (2013). Cochrane Consumers and Communication Review Group: data synthesis and analysis. Cochrane Consumers and Communication Review Group. Retrieved from https://cccrg.cochrane.org/sites/cccrg.cochrane.org/files/public/uploads/Equity.pdf

Sanders, M. R., Kirby, J. N., Tellegen, C. L., \& Day, J. J. (2014). The Triple P-Positive Parenting Program: a systematic review and meta-analysis of a multi-level system of parenting support. Clinical Psychology Review, 34, 337-357. 
654

655

656

657

658

659

660

661

662

663

664

665

666

667

668

669

670

671

672

673

674

675

676

677

678

679

Schomerus, G., Schwahn, C., Holzinger, A., Corrigan, P. W., Grabe, H. J., Carta, M. G., \& Angermeyer, M. C. (2012). Evolution of public attitudes about mental illness: a systematic review and meta-analysis. Acta Psychiatrica Scandinavica, 125, 440-452.

*Shah, R., Draycott, S., Wolpert, M., Christie, D., \& Stein, S. M. (2004). A comparison of Pakistani and Caucasian mothers' perceptions of child and adolescent mental health problems. Emotional and Behavioural Difficulties, 9, 181-190.

Sherman, L. J., \& Ali, M. M. (2017). Mothers' mental health treatment associated with greater adolescent mental health service use for depression. Journal of Child and Family Studies, 26, 2762-2771

Thomas, J., Harden, A., \& Newman, M. (2012). Synthesis: combining results systematically and appropriately. In Gough, D., Oliver, S., \& Thomas, J. (Eds.), An introduction to systematic reviews (pp. 179-226). London, UK: Sage

Turner, E. A., \& Liew, J. (2010). Children's adjustment and child mental health service use: The role of parents' attitudes and personal service use in an upper middle class sample. Community Mental Health Journal, 46, 231-240.

*Umpierre, M., Meyers, L. V., Ortiz, A., Paulino, A., Rodriguez, A. R., Miranda, A., ... \& McKay, M. M. (2015). Understanding Latino parents' child mental health literacy: Todos a bordo/all aboard. Research on Social Work Practice, 25(5), 607-618.

Weed, M., Coren, E., \& Fiore, J. (2009). A systematic review of the evidence base for developing a 24 physical activity and health legacy from the London 2012 Olympic and Paralympics Games. London: Department of Health

*Yap, M. B. H., \& Jorm, A. F. (2012). Parents' beliefs about actions they can take to prevent depressive disorders in young people: results from an Australian national survey. Epidemiology and Psychiatric Sciences, 21, 117-123.

*York, W., \& Jones, J. (2017). Addressing the mental health needs of looked after children in foster care: the experiences of foster carers. Journal of Psychiatric and Mental Health Nursing, 24, 143-153. 
Figure 1

Flow diagram for database search and record screening
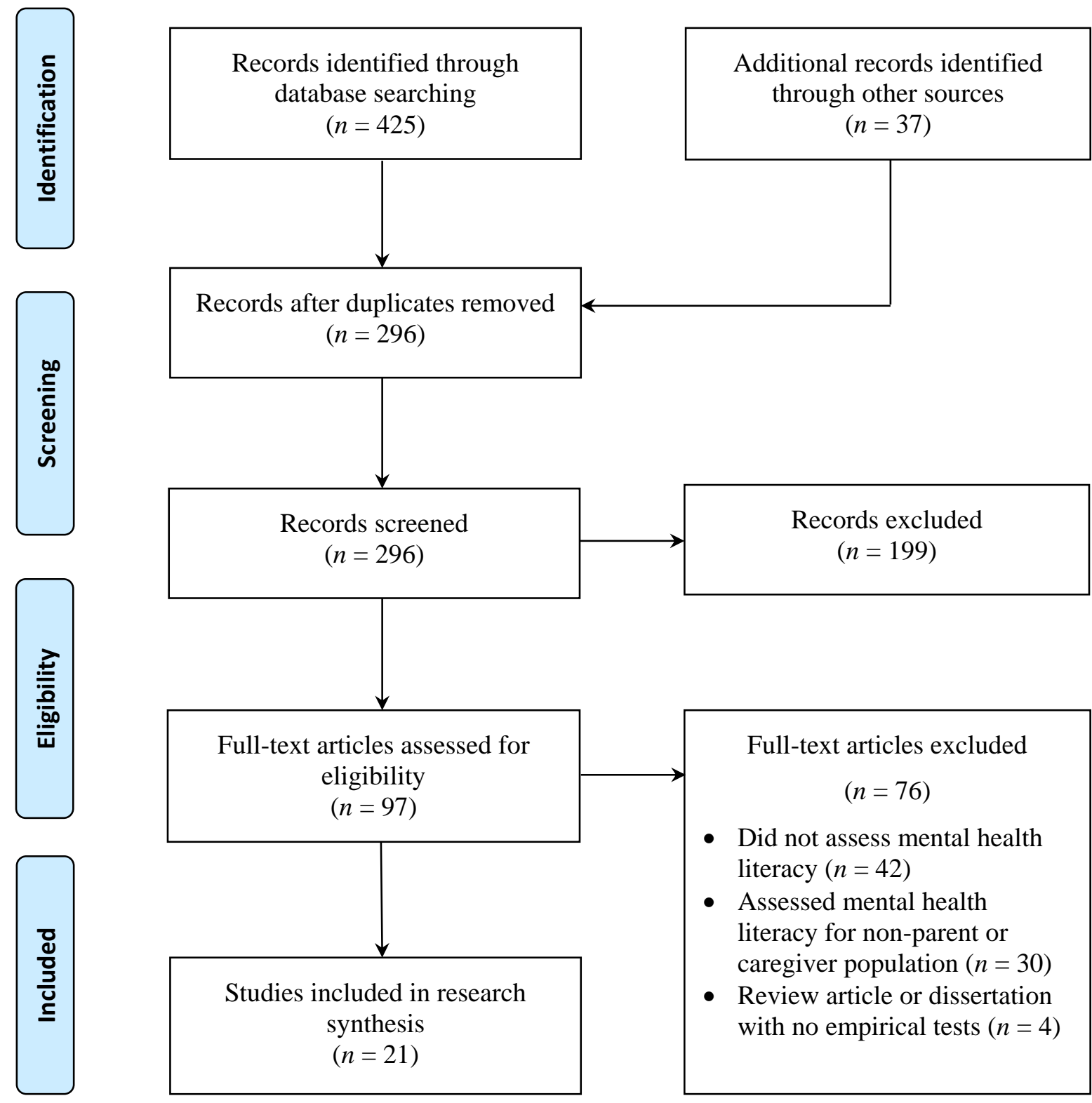
Table 1

Characteristics of included studies

Study Sample demographics

Design

Mental health

Main outcome measures/thematic

Main Findings

Study quality

Quantitative (association) studies

Bonfield et al. (2010)

113 foster car

Cross sectional

In treatment

95 women)

Mendenhall \&

Frauenholtz (2015)

39 parents ( 2 men, 37

Cross sectional

In treatment

women)

\section{Belief in perceived need for}

\section{treatment $(\mathrm{CCI})$}

Knowledge about treatment (TBQ)

Knowledge and understanding of mood disorders (UMDQ)

Parent and child mental health status

Treatment beliefs about the helpfulness or harmfulness of different interventions (SM)

1381 women)

2005 parents $(624 \mathrm{men}$

Cross sectional

Mixed

Beliefs about the helpfulness or
Carers' mental health problem

Attitudes toward seeking professiona

help (ATSPPH)

Carers' help-seeking practices*

Mental health service providers'

perceptions of parental mental health

knowledge (SM)

Perceived accuracy of mental health

knowledge (SM)

Perceived sources of and influences on

parental knowledge (SM) and service use but not actual service use. harmfulness of mental health first aid strategies (SM)
Foster carers were accurate in recognising mental health problems from a vignette but this ability was not linked to problem detection and perceived need for treatment in their own looked after child. Foster carers' favourable attitudes to help-seeking were associated with the increased likelihood of making contact with and referral to mental health services

Providers reported parent mental health literacy as low and mostly inaccurate. Informal sources of support were perceived as the primary source of information for parental mental health knowledge. Having another family member with a mental health disorder, prior treatment experience, and higher level of education were associated with greater perceived parent mental health literacy.

Greater parental knowledge of mood disorders was associated with child bipolar diagnosis, higher level of assuct having educthen having older child health diagnosis and greater number of mental health services currently being utilised. Greater knowledge of treatment was associated with child bipolar diagnosis, greater perceived need for child treatment, being female, being white, and greater number of mental health services being utilised currently.

Parents rated general and informal sources of help (e.g. GP's, family) as more helpful than specialist mental health services. Parents rated dealing with the problem alone, psychiatric medications and hospitalisation negatively.

Parents rated the following first aid strategies as most

Moderate helpful: listening to the person's problems in an understanding way, suggesting they seek professional help, more physically active. Parents were more likely to rate 


\begin{tabular}{|c|c|c|c|c|}
\hline Jorm et al. (2007a) & $\begin{array}{l}2005 \text { parents ( } 624 \text { men, } \\
1381 \text { women) }\end{array}$ & Cross sectional & Mixed & $\begin{array}{l}\text { Help seeking intentions (SM) } \\
\text { Barriers to seeking help (SM) }\end{array}$ \\
\hline Jorm et al. (2007b) & $\begin{array}{l}2005 \text { parents ( } 624 \text { men, } \\
1381 \text { women) }\end{array}$ & Cross sectional & Mixed & $\begin{array}{l}\text { Parents endorsement of mental health } \\
\text { first aid strategies for their child (SM) } \\
\text { Confidence in providing first aid to } \\
\text { child (SM) }\end{array}$ \\
\hline Lawrence et al. (2015) & 6310 parents and carers & Cross sectional & Mixed & $\begin{array}{l}\text { Severity of disorder (SM) } \\
\text { Perceived need for services (SM) } \\
\text { Barriers to service use (SM) }\end{array}$ \\
\hline Shah et al. (2004) & $\begin{array}{l}79 \text { Caucasian and Pakistani } \\
\text { mothers }\end{array}$ & Cross sectional & Prevention & $\begin{array}{l}\text { Problem recognition(SM) } \\
\text { Intention to seek help(SM) }\end{array}$ \\
\hline Yap \& Jorm (2012) & $\begin{array}{l}982 \text { parents ( } 311 \text { men, } 671 \\
\text { women) }\end{array}$ & Cross sectional & Mixed & $\begin{array}{l}\text { Prevention beliefs }(\mathrm{SM}) \\
\text { Correlates of prevention beliefs** }\end{array}$ \\
\hline
\end{tabular}

\section{Quantitative (experimental) studies}

Deitz et al. (2009)

99 parents (54 men, 45 women)

Controlled trial

Mixed

Lopez et al. (2009)
Parent knowledge of depression, Attitudes About Seeking Professiona Psychological Help (ATSPPH) Treatment seeking self-efficacy and confidence (SM)

Symptom identification (SM) Efficacy beliefs in identifying mental illness (SM) anxiety, treatment and parenting (SM)
42 caregivers of relative
with schizophrenia, 63 with schizophrenia, 63
community residents

talking about suicide as harmful rather than helpful.

Parents most frequently endorsed GP's as the intended source of help for their children. The main help-seeking barriers for parents were resistance from the child and cost of treatment.

The most frequently intended mental health first aid actions of parents were to get professional help and

of parents were to get professional help and listen/talk/support person. GP/doctor/medical and
were the most frequent types of professional help were the most frequent types of professional help recommended. Parents reported more confidence
younger children than in helping older children.

Increased severity of the child or adolescent mental health disorder was associated with an increase in parents

perceived need for help. The most commonly identified

barriers were child resistance $(48.4 \%)$, not being sure where to get help (39.6\%), not being able to afford help $(37.0 \%)$, preferring to handle the problem by themselves or with help from family or friends $(31.1 \%)$, and thot sure if alolescent needed help (27\%).

High levels of problem recognition accuracy in both Caucasian and Pakistani mothers. Pakistani mothers were less likely to seek professional help in comparison to Caucasian mothers.

Tertiary education, speaking English, exposure to mental health information at work, and mental health advertising were associated with accurate mental health disorder prevention beliefs in parents. No association was found between parent sex, or parent mental health history and prevention beliefs.

Parents receiving a multimedia web-based mental health literacy program improved their knowledge of children's mental health, mental illness and help-seeking, and their confidence in addressing mental health issues, compared to a no-treatment control condition. No significant differences were found between the groups on help-seeking attitudes.

Community residents and caregivers receiving a brief mental health literacy intervention had increased knowledge of symptoms and greater confidence to identify mental
Moderate

Moderate

Moderate 
Mosuro et al. (2014)

10 foster parents (1 man, $9 \quad$ Uncontrolled trial

Mixed

women)

\section{Qualitative studies}

Honey et al. (2015)

Hurley et al. (2017)

Jeong et al. (2017)

14 parents ( 4 male, 10 female)

32 parents ( 9 men, 23

women)

women) parents (14 men, 32

Focus group

Mixed

Experience and exposure to mental

health.

Dealing with mental health disorders.

Misconceptions about the causes and treatment of depression

Views of depressive symptoms

Beliefs about barriers to treatment Lack of awareness of intervention pathways

Mohamad et al. (2012)

24 caregivers of relatives with schizophrenia (10 men, 14 women)

\section{Montgomery \& Terrion (2016)}

7 new immigrant mothers

Umpierre et al. (2015)
Focus group

Interview

Knowledge about mental illness.

Sources of information.

Coping strategies.

\section{Prevention Mother as communicator.}

Informed views and support of menta

health.

Myths and illusions of mental illness.

In treatment
36 parents and caregivers $\quad$ Focus group (6 men, 30 women)
Attributions to mental illness (SM) Recommended help-seeking options (SM)

oping skills (F/COPES)

Mental Health Awareness (SM)

Causes and effects of mental illness.

illness. Community residents attributed behaviour more to psychosis and were more likely to recommend professiona help-seeking with no change found among caregivers.

The mental health awareness of foster carers was higher following a counselling skills intervention. No significan impact was found on coping skills.

Parent's help-seeking practices were shaped by interacting factors related to their knowledge and beliefs about support strategies and help-seeking options and their ability to apply that knowledge.

Parents had limited mental health literacy, were worried about their adolescents developing a mental health disorder, and wanted information and education on mental health and
help-seeking. Past mental health experience was associated with more favourable attitudes toward mental health and with more favour
help-seeking.

Korean-American parents had limited knowledge and held some misconceptions about depression and its treatment. Parenting style was seen as a primary cause of depression. Fathers appeared to have less knowledge than mothers but ultimately made the final decision on seeking help. Barriers to seeking help included availability, negative beliefs about effectiveness and cost.

Caregivers learned about mental health primarily through interaction with mental health professionals. They had

facilitative attitudes towards mental health and help-seeking but also relied on religious and cultural practices in understanding and treating mental health disorders.

Mothers viewed themselves as being able to communicate about and support youth mental health. They were somewhat informed about mental health but still held stigmatising attitudes.

Parents had fear and suspicion of mental health services and did not have much knowledge until they entered into care. Informal sources of support were important for advice and support. Parents were open to receiving mental health

practices and problems.

Preferred health communication

methods.
Moderate

Moderate

Moderate

Moderate

Moderate

Moderate

Pathways into child mental health care. information. 
Interview

Mixed

Foster carers' psychological understanding of challenging behaviour.

Barriers to accessing mental health

services.

Importance of support

Foster carers had good mental health literacy, and had

experience of mental health services but experienced

barriers within the treatment system. Informal and formal

support networks were important in being able to fulfil foster carer role.

\title{
Mixed-method studies
}

Hurley et al. (2018)

$$
66 \text { parents (17 men, } 51
$$

Controlled Trial

Mixed

Depression Literacy (D-LIT)

Anxiety Literacy (A-LIT)

Attitudes to facilitate mental health

promotion and help seeking (MHLS)

Knowledge of help-seeking options

MHLS)

Confidence to asist (SM)

Parents receiving a brief mental health literacy intervention through community sport clubs improved their knowledge

of depression and anxiety, help-seeking options and

confidence to assist someone experiencing a mental healt disorder compared to a no-treatment control condition. No significant differences were found for parent attitudes.

High

Moderate

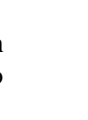

\begin{abstract}
Note. $(\mathrm{SM})=$ measure developed for study. A-LIT = Anxiety Literacy questionnaire (Gulliver et al., 2012), ATSPPH = Attitudes toward seeking professional help (Fischer and Farina, 1995), CCI = Caregiver
concordance interview (Goldberg-Arnold et al., 1999), D-LIT = Depression Literacy questionnaire (Griffiths et al., 2004, F/COPES = The family crisis oriented personal evaluation scales (McCubbin et al., 1991),

MHLS = Mental health literacy scale (O’Connor \& Casey, 2015), TBQ = Treatment beliefs questionnaire (Davidson \& Fristad, 2006), UMDQ = Understanding of mood disorders questionnaire (Gavazzi et al., 1997).
\end{abstract}

*Carers' help-seeking practices $=$ problem detection, perceived need for mental health services, contact with mental health provider, referral to mental health services and use of mental health services, $* *$ Correlates of prevention beliefs $=$ child and parent socio-demographic variables, parent exposure to mental health disorders and information. 
Table 2

Mental health literacy categories and sub-themes

Sub-themes

Mental Health Literacy Categories

Degree and nature of knowledge

Sources of knowledge

Knowledge and Understanding

Correlates of knowledge

Stigma

Role of parent

Attitudes to help-seeking

Attitudes and Beliefs

Fear and worry

Sources of support

Help-seeking strategies

Help-seeking

Factors influencing help-seeking

Controlled trials

Non-controlled trials

Mental Health Literacy Interventions 